\title{
Le jeu en vaut la chandelle
}

\section{Magdalena Seibl}

Lic. phil. I, MAS en travail social, Centre de compétences pour les handicaps visuels et auditifs à un âge avancé

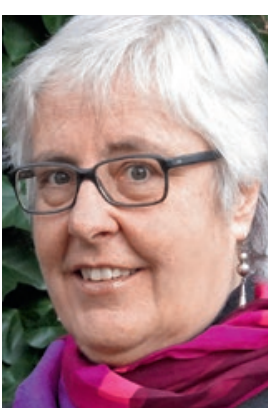

Une amie, dont la mère octogénaire vit en maison de retraite et souffre de dégénérescence maculaire avancée liée à l'âge (DMLA) m'a raconté combien cela l'avait aidée que je lui parle du syndrome de Charles Bonnet. Un jour en faisant les courses, sa maman, soudain hésitante, s'est agrippée à son bras en disant qu'il y avait des trous dans la chaussée, alors qu'il n'y en avait pas. Mon amie me dit avoir pensé à des signes de démence, d'autant plus que sa mère perd la mémoire depuis un certain temps déjà. Elle hésitait à dire à sa mère qu'il n'y avait pas de trou, car elle savait que cette dernière a très peur de souffrir de démence. Elle lui a donc expliqué pour la calmer que de telles hallucinations visuelles sont fréquentes chez les personnes atteintes de déficience visuelle et que cela correspond à une douleur fantôme qui n'a rien à voir avec des signes de démence ou de psychose. Le personnel de la maison de retraite ne connaissait pas ces symptômes.

Dans nos formations sur les troubles visuels et auditifs dans les établissements de soins de longue durée pour les personnes du troisième et du quatrième âge, nous invitons les soignants à motiver les personnes touchées à prendre leur handicap en main et même à envisager une rééducation. Ces personnes et leurs proches ne sont souvent pas conscientes que leur trouble visuel peut avoir des conséquences négatives directes ou indirectes sur leur santé, tant au niveau cognitif, émotionnel, fonctionnel et psychosocial que sur les soins qui leur sont prodigués. De plus, il est fréquent que les personnes âgées taisent leurs difficultés de peur de devenir un poids pour leur entourage ou d'occasionner des coûts supplémentaires. Ainsi, elles renoncent à consulter un ophtalmologue ou à prendre des mesures rééducatives, car « cela n'en vaut plus la peine ».

La situation devient critique lorsque les proches amplifient encore les doutes. La meilleure manière de les soutenir étant de les convaincre que la situation peut être améliorée même à un âge avancé et même si les traitements médicaux sont limités. On peut en effet apprendre à gérer son handicap et à conserver une grande part de son autonomie tout en reconnaissant qu'il est très difficile pour une personne de perdre la vue et que cela lui complique la vie.
L'autonomie et l'état général des personnes atteintes dans leur vision peuvent être clairement améliorés par des exercices ciblés et des adaptations dans leur environnement. Une patiente qui a recours aux soins à domicile cuisine par exemple à nouveau toute seule depuis qu'elle a appris à lire les marques tactiles apposées sur les boutons de sa cuisinière.

Il est possible de rompre le cycle d'effets négatifs par des mesures simples. Si le handicap visuel fait que la personne ne se sent plus en sécurité hors de son domicile, a de la peine à s'orienter et que cela impacte ses contacts sociaux, la rend dépressive et qu'elle s'isole de plus en plus en réduisant ses mouvements et ses activités, cela aura également des conséquences sur son système cardio-vasculaire, son équilibre, ses performances cognitives et sa qualité de vie. Des aides pour s'orienter et un entraînement de la mobilité permettent d'obtenir des améliorations dans tous ces domaines. Les services d'aides aux personnes atteintes de déficience visuelle ou des soignants formés du domaine des soins de longue durée ambulatoires et hospitaliers peuvent épauler ces personnes dans leur apprentissage et dans l'organisation du quotidien.

Il n'est pas facile de rester actif et d'apprendre de nouvelles choses quand on est limité au niveau sensoriel, cela demande passablement d'efforts, mais permet aussi d'améliorer ses facultés cognitives tout en ayant un impact positif sur le vieillissement. L'entraînement et la prise de mesures sociales sont souvent plus efficaces que des aides purement techniques.

Un autre exemple pour s'en convaincre? Un homme de 82 ans atteint de déficience visuelle est admis dans un EMS. Il ne quitte plus sa chambre, passe son temps au lit et refuse catégoriquement de participer à la vie sociale de l'établissement. Pour lui, la vie n'a plus de sens. Il ne répond pas aux tentatives des soignants pour le motiver, mais ceux-ci n'abdiquent pas. Six mois plus tard, il change d'avis et participe peu à peu aux activités de groupe, va manger avec les autres, commence la rééducation spécifique aux troubles visuels et tombe amoureux d'une résidente avec laquelle il va emménager par la suite. Alors, est-ce que le jeu en vaut la chandelle? La réponse est oui, sans hésiter. 